\title{
Resolution of ferrocene and deuterated ferrocene conformations using dynamic vibrational IR spectroscopy
}

\author{
N. T. T. Tran', R. M. Trevorah"1, C. T. Chantler ${ }^{1}$, D. R. T. Appadoo ${ }^{2}$, F. Wang ${ }^{3}$ \\ ${ }^{1}$ School of Physics, The University of Melbourne, Parkville, Victoria 3010, Australia, \\ ${ }^{2}$ Australian Synchrotron, 800 Blackburn Rd, Clayton Victoria 3168, Australia, \\ ${ }^{3}$ Department of Chemistry and Biotechnology, Swinburne University of Technology, Hawthorn, Victoria 3122, Australia
}

nicholast2@student.unimelb.edu.au

The signature of molecular vibrations and distortions in dynamic molecules gives a complex fingerprint which is insightful and can substantiate chemical hypotheses regarding molecular and conformer stability. Using high-accuracy experimental data of ferrocene $(\mathrm{Fc})$ and deuterated ferrocene $\left(\mathrm{dFc}, \mathrm{Fc}-\mathrm{d}^{10}\right)$ at temperatures from $7 \mathrm{~K}$ through to $388 \mathrm{~K}$, we obtain complex spectral profiles which require an advanced reaction coordinate model to explain [1]. We obtain compelling evidence that the single conformer model (staggered $\mathrm{D}_{5 \mathrm{~d}}$ or eclipsed $\mathrm{D}_{5 \mathrm{~h}}$ ) used to interpret and explain many experimental results on ferrocene is invalid. We also present compelling evidence that mixed conformer models are invalid, where ferrocene is represented by an effective dihedral angle between the cyclopentadienyl $(\mathrm{Cp})$ rings; or by a mixture of Boltzmann populations of the two conformers. We find no evidence for single or mixed conformer models despite covering almost all conclusions from past literature for gas, solution or solid phase Fc [1].

A new principle based on the reaction coordinate is introduced using advanced spectroscopy and modelling for hypothesis testing, to articulate the nature of the potential surface, the reaction coordinate, and subtle conformational changes in dilute systems [1]. Theoretical calculations of the infrared spectra of $\mathrm{D}_{5 \mathrm{~h}}$ and $\mathrm{D}_{5 \mathrm{~d}}$ with the $\mathrm{B} 3 \mathrm{LYP} / \mathrm{m} 6-31 \mathrm{G}(\mathrm{d})$ functional highlights a significant difference between Fc conformations around $450-500 \mathrm{~cm}^{-1}$ [2] and early investigations provided key insight into the quantum dynamics of ferrocene [3]. A new methodology for obtaining defined uncertainties with high quality Fourier Transform Infrared (FTIR) measurements allow for quantitative hypothesis testing [4] for complex structural determination. Our experimental analysis shows that the lowest energy conformer is $\mathrm{D}_{5 \mathrm{~h}}$ for both $\mathrm{Fc}$ and $\mathrm{dFc}$ at low temperatures, but as temperature increases, the population of occupied vibrational modes increases towards the $\mathrm{D}_{5 \mathrm{~d}}$ conformation [1]. We obtain agreement of the model with the complex spectral evolution of profiles. These new techniques are sensitive discriminants of alternate models and chemical systems, which argues for wider application to other complex or impenetrable problems across fields arising for numerous other solutions, frozen or at room temperature.

[1] Trevorah, R.M., Tran, N.T.T., Appadoo, D.R.T., Wang, F., Chantler, C.T. (2020). Inorganica Chimica Acta, 506, 119491

[2] Mohammadi, N., Ganesan, A., Chantler C.T., Wang, F. (2012). Journal of Organometallic Chemistry. 713, 51-59

[3] Best, S.P., Wang, F., Islam, M.T., Islam, S., Appadoo, D., Trevorah, R.M., Chantler, C.T. (2016). Chemistry - A European Journal. 22 (50), $18019-18026$

[4] Islam, M.T., Trevorah, R.M., Appadoo, D.R.T., Best, S.P., Chantler, C.T. (2017). Spectrochimica Acta -Part A: Molecular and Biomolecular Spectroscopy, 177, 86-92

Keywords: Infrared spectroscopy; Stereochemical analysis; Ferrocene; High-resolution FTIR

This research is supported by the AINSE Honours Scholarship Program. 